\title{
APPROXIMATION BY ANALYTIC OPERATOR FUNCTIONS. FACTORIZATIONS AND VERY BADLY APPROXIMABLE FUNCTIONS
}

\author{
V. V. PELLER AND S. R. TREIL
}

\begin{abstract}
This is a continuation of our earlier paper published in Selecta Math 11 (2005), 127-154. We consider here operator-valued functions (or infinite matrix functions) on the unit circle $\mathbb{T}$ and study the problem of approximation by bounded analytic operator functions. We discuss thematic and canonical factorizations of operator functions and study badly approximable and very badly approximable operator functions.

We obtain algebraic and geometric characterizations of badly approximable and very badly approximable operator functions. Note that there is an important difference between the case of finite matrix functions and the case of operator functions. Our criteria for a function to be very badly approximable in the case of finite matrix functions also guarantee that the zero function is the only superoptimal approximant. However, in the case of operator functions this is not true.
\end{abstract}

\section{$\S 1$. INTRODUCTION}

Our previous paper PT3 was devoted to a characterization of very badly approximable matrix functions. In this paper we consider the case of operator-valued functions or, which is equivalent, infinite matrix functions.

Background (best approximation and badly approximable functions). The classical problem of analytic approximation for a given bounded function $\varphi$ on the unit circle $\mathbb{T}$ is to find a function $f$ in the Hardy class $H^{\infty}$ such that

$$
\|\varphi-f\|_{\infty}=\operatorname{dist}_{L^{\infty}}\left(\varphi, H^{\infty}\right)=\inf _{h \in H^{\infty}}\|\varphi-h\|_{\infty} .
$$

Such a best approximant $f$ always exists (a compactness argument); S. Khavinson [Kh] proved that it is unique if $\varphi$ is continuous.

A function $\varphi \in L^{\infty}$ is said to be badly approximable if

$$
\|\varphi\|_{\infty}=\operatorname{dist}_{L^{\infty}}\left(\varphi, H^{\infty}\right) .
$$

There is an elegant characterization of the set of continuous badly approximable functions: a nonzero continuous function $\varphi$ on $\mathbb{T}$ is badly approximable if and only if it has constant modulus and its winding number wind $\varphi$ with respect to the origin is negative AAK, $\mathrm{Po}$.

It is well known that this criterion can be extended to a broader class of functions $\varphi$, for which the winding number is not defined. To do this, we need the notions of Hankel

2000 Mathematics Subject Classification. Primary 30D55, 47S35, 30E10, 46E40.

Key words and phrases. Superoptimal approximation, badly approximable operator functions, very badly approximable operator functions, Hankel operators, Toeplitz operators.

The first author was partially supported by NSF grant DMS 0200712. The second author was partially supported by NSF grant DMS 0200584. 
and Toeplitz operators. The Toeplitz operator $T_{\varphi}: H^{2} \rightarrow H^{2}$ and the Hankel operator $H_{\varphi}: H^{2} \rightarrow H_{-}^{2} \stackrel{\text { def }}{=} L^{2} \ominus H^{2}$ are defined by

$$
T_{\varphi} f=\mathbb{P}_{+} \varphi f, \quad H_{\varphi} f=\mathbb{P}_{-} \varphi f,
$$

where $\mathbb{P}_{-}$and $\mathbb{P}_{+}$are the orthogonal projections onto the subspaces $H^{2}$ and $H_{-}^{2} \stackrel{\text { def }}{=}$ $L^{2} \ominus H^{2}$ of $L^{2}$.

It is well known (see, e.g., $\mathrm{D}$ or $[\mathrm{Pe} 2]$ ) that if $\varphi \in C(\mathbb{T})$ and $\varphi$ does not vanish on $\mathbb{T}$, then the Toeplitz operator $T_{\varphi}$ on the Hardy class $H^{2}$ is Fredholm, and ind $T_{\varphi}=-$ wind $\varphi$ (recall that for a Fredholm operator $A$ its index is defined as ind $A=\operatorname{dim} \operatorname{Ker} A-$ $\operatorname{dim} \operatorname{Ker} A^{*}$ ). The above characterization of badly approximable functions can easily be generalized in the following way: if $\varphi$ is a function in $L^{\infty}$ such that the essential norm $\left\|H_{\varphi}\right\|_{\mathrm{e}}$ of the Hankel operator $H_{\varphi}$ (i.e., the distance from $H_{\varphi}$ to the set of compact operators) is less than its norm, then $\varphi$ is badly approximable if and only if $\varphi$ has constant modulus almost everywhere on $\mathbb{T}, T_{\varphi}$ is Fredholm, and ind $T_{\varphi}>0$ (see, e.g., $[$ Pe2, Chapter 7, §5]). Also, we recall that

$$
\left\|H_{\varphi}\right\|=\operatorname{dist}_{L^{\infty}}\left(\varphi, H^{\infty}\right) \quad \text { and } \quad\left\|H_{\varphi}\right\|_{\mathrm{e}}=\operatorname{dist}_{L^{\infty}}\left(\varphi, H^{\infty}+C\right)
$$

(see, e.g., [Pe2]).

Let us proceed now to the case of matrix functions. We can consider the same problem of finding a best analytic approximant for a given bounded function $\Phi$ with values in the space $\mathbb{M}_{m, n}$ of $m \times n$ matrices: for $\Phi \in L^{\infty}\left(\mathbb{M}_{m, n}\right)$ find a bounded analytic $\mathbb{M}_{m, n}$-valued function $F$ such that

$$
\|\Phi-F\|_{L^{\infty}}=\operatorname{dist}_{L^{\infty}\left(\mathbb{M}_{m, n}\right)}\left(\Phi, H^{\infty}\left(\mathbb{M}_{m, n}\right)\right) .
$$

Here

$$
\|\Phi\|_{L^{\infty}} \stackrel{\text { def }}{=} \underset{\zeta \in \mathbb{T}}{\operatorname{ess~sup}}\|\Phi(\zeta)\|_{\mathbb{M}_{m, n}}
$$

$\mathbb{M}_{m, n}$ is equipped with the standard operator norm, and $H^{\infty}\left(\mathbb{M}_{m, n}\right)$ is the space of bounded analytic functions with values in $\mathbb{M}_{m, n}$.

Again, it can easily be shown that a best approximant always exists. However, the situation with uniqueness is quite different from the scalar case. Indeed, suppose that $m=n=2$ and $u$ is a scalar continuous badly approximable unimodular function (i.e., $|u(\zeta)|=1$ almost everywhere on $\mathbb{T})$. Consider the matrix function $\Phi=\left(\begin{array}{ll}u & 0 \\ 0 & 0\end{array}\right)$. It is easy to check that for any scalar function $f$ in the unit ball of $H^{\infty}$, the matrix function $\left(\begin{array}{ll}0 & 0 \\ 0 & f\end{array}\right)$ is a best approximation of $\Phi$.

While it is possible to describe badly approximable matrix- and operator-valued functions, and we give such descriptions in this paper (the case of finite matrix functions was treated in our earlier paper [PT3]), this is not our main goal. It turns out that in the matrix case it is more natural to consider superoptimal approximations and very badly approximable functions.

Superoptimal approximations and very badly approximable matrix functions. Recall that, for a matrix (or a bounded linear operator on Hilbert space) $A$, the singular values $s_{j}(A), j \geq 0$, are defined by

$$
s_{j}(A)=\inf \{\|A-K\|: \operatorname{rank} K \leq j\} .
$$

Clearly, $s_{0}(A)=\|A\|$. 
Definition. Given a matrix function $\Phi \in L^{\infty}\left(\mathbb{M}_{m, n}\right)$, we introduce inductively the sets $\boldsymbol{\Omega}_{j}, 0 \leq j \leq \min \{m, n\}-1$, by

$$
\begin{aligned}
& \boldsymbol{\Omega}_{0}=\left\{F \in H^{\infty}\left(\mathbb{M}_{m, n}\right): F \text { minimizes } t_{0} \stackrel{\text { def }}{=} \underset{\zeta \in \mathbb{T}}{\operatorname{ess~sup}}\|\Phi(\zeta)-F(\zeta)\|\right\} ; \\
& \boldsymbol{\Omega}_{j}=\left\{F \in \Omega_{j-1}: F \text { minimizes } t_{j} \stackrel{\text { def }}{=} \underset{\zeta \in \mathbb{T}}{\operatorname{ess} \sup } s_{j}(\Phi(\zeta)-F(\zeta))\right\}, \quad j>0 .
\end{aligned}
$$

The functions in $\bigcap_{k \geq 0} \boldsymbol{\Omega}_{k}=\boldsymbol{\Omega}_{\min \{m, n\}-1}$ are called superoptimal approximants of $\Phi$ by bounded analytic matrix functions. The numbers $t_{j}=t_{j}(\Phi)$ are called the superoptimal singular values of $\Phi$. Note that the functions in $\boldsymbol{\Omega}_{0}$ are precisely the best approximants by bounded analytic matrix functions.

As in the case of scalar functions, a bounded $m \times n$ matrix function $\Phi$ is said to be badly approximable if

$$
\|\Phi\|_{L^{\infty}}=\inf \left\{\|\Phi-F\|_{L^{\infty}}: F \in H^{\infty}\left(\mathbb{M}_{m, n}\right)\right\} .
$$

We say that a matrix function $\Phi \in L^{\infty}\left(\mathbb{M}_{m, n}\right)$ is very badly approximable if the zero function $\mathbf{0}$ is a superoptimal approximant of $\Phi$.

The notion of superoptimal approximation can be extended to the case of operatorvalued functions. If $\mathcal{H}$ and $\mathcal{K}$ are Hilbert spaces, we denote by $\mathcal{B}(\mathcal{H}, \mathcal{K})$ the space of bounded linear operators from $\mathcal{H}$ to $\mathcal{K}$,

$$
\mathcal{B}(\mathcal{H}) \stackrel{\text { def }}{=} \mathcal{B}(\mathcal{H}, \mathcal{H})
$$

We can identify an infinite-dimensional separable Hilbert space with $\ell^{2}$ and identify operators on $\ell^{2}$ with infinite matrices. Suppose that $\Phi \in L^{\infty}\left(\mathcal{B}\left(\ell^{2}\right)\right)$, i.e., $\Phi$ is a weakly measurable bounded function that takes values in $\mathcal{B}\left(\ell^{2}\right)$. Then we can define a sequence $\left\{\boldsymbol{\Omega}_{j}\right\}_{j \geq 0}$ in the same way as for finite matrix functions. However, in the case of operator-valued functions we must consider the infinite sequence of the sets $\boldsymbol{\Omega}_{j}$. For $\Phi \in L^{\infty}\left(\mathcal{B}\left(\ell^{2}\right)\right)$, we say that a function $F$ in $H^{\infty}\left(\mathcal{B}\left(\ell^{2}\right)\right)$ is a superoptimal approximant of $\Phi$ by bounded analytic operator functions if $F \in \bigcap_{j \geq 0} \boldsymbol{\Omega}_{j}$.

Badly approximable and very badly approximable infinite matrix functions can be defined in the same way as in the case of finite matrix functions.

Note that if $\Phi$ is a matrix function of size $m \times \infty$ or $\infty \times n$, we can add to $\Phi$ infinitely many zero rows or zero columns and reduce the problem to the case of matrix functions of size $\infty \times \infty$.

The summary of earlier results. First of all, we mention that superoptimal approximation is more natural in the case of matrix or operator functions, because it is unique under mild and natural assumptions on the function. It was shown in PY1 that if $\Phi \in\left(H^{\infty}+C\right)\left(\mathbb{M}_{m, n}\right)$ (i.e., all entries of $\Phi$ belong to $\left.H^{\infty}+C\right)$, then $\Phi$ has a unique superoptimal approximation $F$ by bounded analytic matrix functions. Moreover, in PY1] it was proved that

$$
s_{j}(\Phi(\zeta)-F(\zeta))=t_{j}(\Phi) \quad \text { for almost all } \zeta \in \mathbb{T} .
$$

Later this result was extended in [T] (see also [P1, PT1]) to operator-valued functions $\Phi$ for which the Hankel operator $H_{\Phi}$ is compact.

The proof given in [PY1] was based on certain special factorizations (thematic factorizations; see $\S 4$ of this paper for definitions). The approach in [T] was more geometric and based on the notion of superoptimal weights.

The problem of describing the very badly approximable functions was posed in PY1]. It follows from (1.2) that if $\Phi$ is a very badly approximable function in $\left(H^{\infty}+C\right)\left(\mathbb{M}_{m, n}\right)$, then the singular values $s_{j}(\Phi(\zeta))$ are constant for almost all $\zeta \in \mathbb{T}$. Moreover, in PY1] 
it was shown that if, in addition to this, $m \leq n$ and $s_{m-1}(\Phi(\zeta)) \neq 0$ almost everywhere, then the Toeplitz operator $T_{z \Phi}: H^{2}\left(\mathbb{C}^{n}\right) \rightarrow H^{2}\left(\mathbb{C}^{m}\right)$ has dense range (if $\Phi$ is a scalar function, the latter condition is equivalent to the fact that ind $\left.T_{\Phi}>0\right)$. Note that the Toeplitz and the Hankel operators whose symbols are matrix functions can be defined in the same way as in the scalar case (see (1.1)). Obviously, this necessary condition is equivalent to the condition $\operatorname{Ker} T_{\bar{z} \Phi^{*}}=\{\mathbf{0}\}$. In fact, the proof of necessity given in PY1 allows one to obtain a more general result: if $\Phi$ is an arbitrary very badly approximable function in $\left(H^{\infty}+C\right)\left(\mathbb{M}_{m, n}\right)$ and $f \in \operatorname{Ker} T_{\bar{z} \Phi^{*}}$, then $\Phi^{*} f=\mathbf{0}$.

On the other hand, in PY1 an example of a continuous $2 \times 2$ function $\Phi$ was given such that $s_{0}(\Phi(\zeta))=1, s_{1}(\Phi(\zeta))=\alpha<1$ for $\zeta \in \mathbb{T}$, and $T_{z \Phi}$ is invertible, but $\Phi$ is not even badly approximable.

The very badly approximable matrix functions of class $\left(H^{\infty}+C\right)\left(\mathbb{M}_{m, n}\right)$ were characterized in [PY1] algebraically, in terms of the so-called thematic factorizations.

Later, in [PT2] the above results of [PY1] were generalized to the broader context of matrix functions $\Phi$ such that the essential norm $\left\|H_{\Phi}\right\|_{\mathrm{e}}$ of the Hankel operator $H_{\Phi}$ is less than the smallest nonzero superoptimal singular value of $\Phi$. We call such $\Phi$ admissible matrix functions. In particular, if $\Phi$ is an admissible very badly approximable $m \times n$ matrix function, then the functions $s_{j}(\Phi(z))$ are constant almost everywhere on $\mathbb{T}$ and

$$
\operatorname{Ker} T_{\bar{z} \Phi^{*}}=\left\{f \in H^{2}\left(\mathbb{C}^{n}\right): \Phi^{*} f=\mathbf{0}\right\} .
$$

In $[\mathrm{AP}$, another algebraic characterization of the set of very badly approximable admissible matrix functions was given in terms of canonical factorizations (see $\S 5$ for the definition).

We refer the reader to the book [Pe2], which contains all the above information and results on superoptimal approximation and very badly approximable functions.

In PT3 we obtained a new criterion for an admissible matrix function to be very badly approximable. While earlier criteria in terms of certain special factorizations are very helpful in constructing very badly approximable functions and studying their properties, the new one is more geometric and it is easier to use it to verify whether a given matrix function is very badly approximable. This criterion was given in terms of families of subspaces spanned by Schmidt vectors of the matrices $\Phi(\zeta), \zeta \in \mathbb{T}$.

Recall that if $A$ is an $m \times n$ matrix and $s$ is a singular value of $A$, then a nonzero vector $x \in \mathbb{C}^{n}$ is called a Schmidt vector corresponding to $s$ if $A^{*} A x=s^{2} x$.

Given a matrix function $\Phi$ in $L^{\infty}\left(\mathbb{M}_{m, n}\right)$ and $\sigma>0$, we considered the subspace $\mathfrak{S}_{\Phi}^{(\sigma)}(\zeta)$ of $\mathbb{C}^{n}$ spanned by the Schmidt vectors of $\Phi(\zeta)$ that correspond to the singular values of $\Phi(\zeta)$ that are greater than or equal to $\sigma$. The subspaces $\mathfrak{S}_{\Phi}^{(\sigma)}(\zeta)$ are defined for almost all $\zeta \in \mathbb{T}$. In [PT3] it was shown that if $\Phi$ is an admissible very badly approximable matrix function, then for each $\sigma>0$ the family of subspaces $\mathfrak{S}_{\Phi}^{(\sigma)}(\zeta), \zeta \in \mathbb{T}$, is analytic, i.e., there exist functions $g_{1}, \ldots, g_{k}$ in $H^{2}\left(\mathbb{C}^{n}\right)$ such that

$$
\mathfrak{S}_{\Phi}^{(\sigma)}(\zeta)=\operatorname{span}\left\{g_{1}(\zeta), \ldots, g_{k}(\zeta)\right\} \quad \text { for almost all } \zeta \in \mathbb{T} .
$$

The same analyticity condition must also be imposed on the transposed function $\Phi^{\mathrm{t}}$. However, as was shown in [PT3, the analyticity conditions on $\Phi$ and $\Phi^{\mathrm{t}}$ together with the earlier necessary conditions quoted above do not guarantee that $\Phi$ is very badly approximable.

Nevertheless, it turns out that the above condition can be slightly modified to get a necessary and sufficient condition. The main result of [PT3] is the following theorem.

Theorem. Let $\Phi$ be an admissible matrix function. Then $\Phi$ is very badly approximable if and only if, for each $\sigma>0$, equality (1.3) holds for some functions $g_{1}, \ldots, g_{k}$ in 
$\operatorname{Ker} T_{\Phi}$. Moreover, this condition implies that $\Phi$ is very badly approximable even without the assumption that $\Phi$ is admissible.

Note that in the case of a scalar function $\varphi$ this condition means that $\varphi$ has constant modulus and $\operatorname{Ker} T_{\varphi} \neq\{\mathbf{0}\}$, i.e., our criterion is a natural generalization of the scalar results discussed above.

The uniqueness problem for superoptimal approximation of operator functions (infinite matrix functions) was studied in [T, Pe1, PT1. It was shown there that if the Hankel operator $H_{\Phi}$ is compact, then $\Phi$ has a unique superoptimal approximant among bounded analytic operator functions. In [Pe1] and [PT1] uniqueness was obtained with the help of partial thematic factorizations (see $\S 4$ of this paper). We also refer the reader to the monograph [Pe2] for the above results on superoptimal approximation of operator functions.

The purpose and outline of the paper. In this paper we study very badly approximable operator functions. We consider the class of admissible operator functions. As in the case of finite matrix functions, an operator function $\Phi$ is said to be admissible if the essential norm $\left\|H_{\Phi}\right\|_{\mathrm{e}}$ of the Hankel operator $H_{\Phi}$ is less than each nonzero superoptimal singular value of $\Phi$.

In $\S 4$ we consider partial thematic factorizations of admissible operator functions (without the assumption of the compactness of $H_{\Phi}$ as was done in [Pe1 and [PT1]). In $\S 5$ we consider partial canonical factorizations of operator functions.

The main result of the paper is a criterion of very bad approximability (Theorem 6.1) presented in $\S 6$. Essentially, it says that the theorem stated above is also true in the case of operator functions.

However, it turns out that there is an important distinction between the case of finite matrix functions and the case of infinite matrix functions. In the case of finite matrix functions, if $\Phi$ satisfies the hypotheses of the above theorem, then the zero function is the only superoptimal approximant of $\Phi$. We show in this paper that in the case of infinite matrix functions this is no longer true: under the hypotheses of the above theorem, $\Phi$ must be very badly approximable, but it can have infinitely many superoptimal approximants.

Note also that in the case of infinite matrix functions some proofs are considerably more complicated than the proofs of the corresponding results for finite matrix functions (see, e.g., the proofs of Theorems 3.1 and 5.1 given below).

In $\S 2$ we define inner, outer, and co-outer operator functions and prove a theorem about inner-outer factorizations of co-outer operator functions.

In $\S 3$ we define balanced operator functions and prove that both inner and co-outer functions with finitely many columns have a balanced completion.

\section{§2. INNER AND OUTER OPERATOR FUNCTIONS}

In this section we define inner, outer, and co-outer operator functions and prove that the inner factor in the inner-outer factorization of a co-outer function with finitely many columns must also be co-outer.

Let $\mathcal{H}$ and $\mathcal{K}$ be separable Hilbert spaces. We denote by $H_{\mathrm{s}}^{2}(\mathcal{B}(\mathcal{H}, \mathcal{K}))$ the space of analytic operator functions $F$ that take values in the space of bounded linear operators from $\mathcal{H}$ to $\mathcal{K}$ and satisfy the following condition:

$$
F(z) x \in H^{2}(\mathcal{K}) \text { for every } x \in \mathcal{H} \text {. }
$$

A function $F$ in $H_{\mathrm{s}}^{2}(\mathcal{B}(\mathcal{H}, \mathcal{K}))$ is said to be inner if $F(\zeta)$ is an isometric operator (i.e., $\left.F(\zeta)^{*} F(\zeta)=I\right)$ for almost all $\zeta \in \mathbb{T}$. A function $F$ in $H_{\mathrm{s}}^{2}(\mathcal{B}(\mathcal{H}, \mathcal{K}))$ is outer if the set

$$
\left\{F q: q \text { is a polynomial in } H^{2}(\mathcal{H})\right\}
$$

is dense in $H^{2}(\mathcal{K})$. 
It is well known (see, e.g., $\mathrm{N}]$ ) that each function $F$ in $H_{\mathrm{s}}^{2}(\mathcal{B}(\mathcal{H}, \mathcal{K}))$ admits an innerouter factorization, i.e., there exists an inner operator function $\Theta$ and an outer operator function $G$ such that $F=\Theta G$.

As mentioned in the Introduction, we are going to identify operator functions with infinite matrix functions. We say that an infinite matrix function $F$ is co-outer if the transposed function $F^{\mathrm{t}}$ is outer.

Theorem 2.1. Let $F$ be a co-outer operator function in $H_{\mathrm{s}}^{2}\left(\mathcal{B}\left(\mathbb{C}^{d}, \ell^{2}\right)\right), d<\infty$. Suppose that $F=\Theta G$, where $\Theta$ is an inner operator function and $G$ is an outer operator function. Then $\Theta$ is co-outer.

Proof. Suppose $\Theta \in H^{\infty}\left(\mathcal{B}\left(\mathbb{C}^{k}, \ell^{2}\right)\right)$ and $G \in H^{2}\left(\mathbb{M}_{d, k}\right)$. Since $G$ is outer, it follows that $k \leq d$. Suppose that $\Theta^{\mathrm{t}}=\mathcal{O} Q$, where $\mathcal{O}$ is an inner matrix function and $Q$ is an outer operator function. Since $\Theta$ is inner, it is easily seen that $\mathcal{O}$ has size $k \times k$. We have $\Theta=Q^{\mathrm{t}} \mathcal{O}^{\mathrm{t}}$. Then $F=Q^{\mathrm{t}} \mathcal{O}^{\mathrm{t}} G$. Therefore, by the hypotheses of the theorem, $F^{\mathrm{t}}=G^{\mathrm{t}} \mathcal{O} Q$ is an outer function. It follows that $G^{\mathrm{t}}$ must be outer, so that $k=d$. Clearly, $G^{\mathrm{t}} \mathcal{O} H^{\infty}\left(\mathbb{M}_{d, d}\right)$ must be dense in $H^{2}\left(\mathbb{M}_{d, d}\right)$. However, the determinants of all matrix functions in $G^{\mathrm{t}} \mathcal{O} H^{\infty}\left(\mathbb{M}_{d, d}\right)$ must be divisible by $\operatorname{det} \mathcal{O}$, which is a scalar inner function. Thus, $\operatorname{det} \mathcal{O}$ is constant, whence $\mathcal{O}^{*}=\mathcal{O}^{-1} \in H^{\infty}\left(\mathbb{M}_{d, d}\right)$. This implies that $\mathcal{O}$ is constant, and so $\Theta$ is co-outer.

\section{$\S 3$. BALANCED MATRIX FUNCTIONS}

In this section we introduce the notion of a balanced unitary-valued function and prove the existence of balanced completions for the inner and co-outer functions that have finitely many columns.

Definition. A balanced infinite matrix function is a unitary-valued matrix function of the form $(\Upsilon \bar{\Theta})$, where $\Upsilon$ and $\Theta$ are inner and co-outer matrix functions.

If $\Upsilon$ has $r$ columns, we say that the function $(\Upsilon \bar{\Theta})$ is $r$-balanced. 1-balanced functions are also called thematic matrix functions.

We are going to prove that any inner matrix function with finitely many columns can be completed to a balanced matrix function.

Let $r$ be a positive integer, and let $\Upsilon$ be an inner matrix function in $H^{\infty}\left(\mathbb{C}^{r}, \ell^{2}\right)$. Consider the subspace $\mathcal{L} \stackrel{\text { def }}{=} \operatorname{Ker} T_{\Upsilon^{t}}$ of $H^{2}\left(\ell^{2}\right)$. Clearly, it is invariant under multiplication by $z$, and so there exists an inner matrix function $\Theta$ such that $\mathcal{L}=\Theta H^{2}(\mathcal{K})$, where $\mathcal{K}=\ell^{2}$ or $\mathcal{K}=\mathbb{C}^{m}$ for some positive $m$. The proof of the following theorem in the special case of $r=1$ can be found in [Pe2, Chapter 14, §18]. In the general case the proof is algebraically more complicated. Note that a close result was obtained in $[\mathrm{C}$; see also [H, Lecture IX].

Theorem 3.1. Let $\Upsilon$ and $\Theta$ be as above. Then $\Theta$ is co-outer and the matrix function $(\Upsilon \bar{\Theta})$ is unitary-valued.

Before proceeding to the proof, we introduce a notion. Let

$$
A=\left(\begin{array}{cccc}
a_{11} & a_{12} & \cdots & a_{1 r} \\
a_{21} & a_{22} & \cdots & a_{2 r} \\
\vdots & \vdots & \ddots & \vdots \\
a_{r+11} & a_{r+12} & \cdots & a_{r+1 r}
\end{array}\right)
$$


be an $(r+1) \times r$ matrix. For $1 \leq j \leq r+1$, we put

$$
\alpha_{j}=(-1)^{j} \operatorname{det}\left(\begin{array}{ccc}
a_{11} & \cdots & a_{1 r} \\
\vdots & \ddots & \vdots \\
a_{j-11} & \cdots & a_{j-1 r} \\
a_{j+11} & \cdots & a_{j+1 r} \\
\vdots & \ddots & \vdots \\
a_{r+11} & \cdots & a_{r+1 r}
\end{array}\right)
$$

In other words, we multiply $(-1)^{j}$ by the minor obtained from $A$ by deleting the $j$ th row. The vector $A_{\text {ass }} \stackrel{\text { def }}{=}\left\{\alpha_{j}\right\}_{1 \leq j \leq r+1}$ is called the vector associated with $A$.

Proof. The proof of the fact that $\Theta$ is co-outer is exactly the same as in the case of $r=1$; see [Pe2, Chapter 14, Lemma 18.3]. Let us show that $(\Upsilon \bar{\Theta})$ is unitary-valued. The fact that $(\Upsilon \bar{\Theta})$ takes isometric values almost everywhere on $\mathbb{T}$ follows immediately from the definition of $\Theta$. To prove that it is unitary-valued, it suffices to show that $\operatorname{dim} \operatorname{Ker} \Theta^{\mathrm{t}}(\zeta) \leq r$ for almost all $\zeta \in \mathbb{T}$.

Let

$$
\Upsilon=\left(\begin{array}{cccc}
v_{01} & v_{02} & \cdots & v_{0 r} \\
v_{11} & v_{12} & \cdots & v_{1 r} \\
v_{21} & v_{22} & \cdots & v_{2 r} \\
\vdots & \vdots & \ddots & \vdots
\end{array}\right)
$$

Clearly, the matrix function $\Upsilon$ has rank $r$ almost everywhere on $\mathbb{T}$. Without loss of generality we may assume that

$$
\operatorname{det}\left(\begin{array}{cccc}
v_{01} & v_{02} & \cdots & v_{0 r} \\
v_{11} & v_{12} & \cdots & v_{1 r} \\
v_{21} & v_{22} & \cdots & v_{2 r} \\
\vdots & \vdots & \ddots & \vdots \\
v_{r-11} & v_{r-12} & \cdots & v_{r-1 r}
\end{array}\right) \neq \mathbf{0}
$$

Consider the bounded analytic matrix function $G$ defined in the following way:

$$
\boldsymbol{G}=\left(\begin{array}{ccccc}
\alpha_{0}^{[0]} & \alpha_{0}^{[1]} & \alpha_{0}^{[2]} & \alpha_{0}^{[3]} & \ldots \\
\alpha_{1}^{[0]} & \alpha_{1}^{[1]} & \alpha_{1}^{[2]} & \alpha_{1}^{[3]} & \ldots \\
\vdots & \vdots & \vdots & \vdots & \ddots \\
\alpha_{r-1}^{[0]} & \alpha_{r-1}^{[1]} & \alpha_{r-1}^{[2]} & \alpha_{r-1}^{[3]} & \ldots \\
\alpha_{r}^{[0]} & \mathbf{0} & \mathbf{0} & \mathbf{0} & \ldots \\
\mathbf{0} & \alpha_{r}^{[0]} & \mathbf{0} & \mathbf{0} & \ldots \\
\mathbf{0} & \mathbf{0} & \alpha_{r}^{[0]} & \mathbf{0} & \ldots \\
\vdots & \vdots & \ddots & \ddots & \ddots
\end{array}\right),
$$


where for $k \geq 0$, the $H^{\infty}$-functions $\alpha_{m}^{[k]}, 0 \leq m \leq r$, are the components of the vector function $A_{\text {ass }}^{[k]}$ associated with the matrix function $A^{[k]}$ defined by

$$
A^{[k]}=\left(\begin{array}{cccc}
v_{01} & v_{02} & \cdots & v_{0 r} \\
v_{11} & v_{12} & \cdots & v_{1 r} \\
v_{21} & v_{22} & \cdots & v_{2 r} \\
\vdots & \vdots & \ddots & \vdots \\
v_{r-11} & v_{r-12} & \cdots & v_{r-1 r} \\
v_{r+k 1} & v_{r+k 2} & \cdots & v_{r+k r}
\end{array}\right) .
$$

Note that $\alpha_{r}^{[0]}$ is nothing but the determinant on the left-hand side of (3.1).

It is an elementary exercise in linear algebra to verify that $\Upsilon^{\mathrm{t}} G=\mathbf{0}$. It follows that $G$ admits a factorization $G=\Theta Q$, where $Q$ is an $H^{\infty}$ matrix function. Hence, to verify that $\operatorname{dim} \operatorname{Ker} \Theta^{\mathrm{t}}(\zeta) \leq r$, it suffices to show that $\operatorname{dim} \operatorname{Ker} G^{\mathrm{t}}(\zeta) \leq r$. Recall that, by (3.1), $\alpha_{r}^{[0]}(\zeta) \neq 0$ for almost all $\zeta \in \mathbb{T}$. Assume that $\zeta \in \mathbb{T}$ and $\alpha_{r}^{[0]}(\zeta) \neq 0$. Suppose that the vector $x=\left\{x_{j}\right\}_{j \geq 0}$ belongs to $\operatorname{Ker} G^{\mathrm{t}}(\zeta)$. If we look at the $r$ th coordinate of the vector $G^{\mathrm{t}}(\zeta) x$, we observe that $\alpha_{r}^{[0]}(\zeta) x_{r}$ is uniquely determined by $x_{0}, x_{1}, \ldots, x_{r-1}$. Since $\alpha_{r}^{[0]}(\zeta) \neq 0$, it follows that $x_{r}$ is uniquely determined by $x_{0}, x_{1}, \ldots, x_{r-1}$. If we look now at the next component of the vector $G^{\mathrm{t}}(\zeta) x$, we observe that $x_{r+1}$ is uniquely determined by $x_{0}, x_{1}, \ldots, x_{r}$, etc. This completes the proof.

\section{$\S 4$. Partial thematic Factorizations}

In the case where $\Phi$ is an operator function such that the Hankel operator $H_{\Phi}$ is compact and $F \in \boldsymbol{\Omega}_{d}$, partial thematic factorizations of $\Phi-F$ were constructed in [Pe1]. In this section we consider the more general case where $\Phi$ is an admissible operator function.

Suppose that $\Phi$ is a function in $L^{\infty}\left(\mathcal{B}\left(\ell^{2}\right)\right)$ such that

$$
\left\|H_{\Phi}\right\|_{\mathrm{e}}<\left\|H_{\Phi}\right\|
$$

and $F \in H^{\infty}\left(\mathcal{B}\left(\ell^{2}\right)\right)$ is a best approximant of $\Phi$. Then $H_{\Phi}$ has a maximizing vector $f$, and the function $g=\left\|H_{\Phi}\right\|^{-1} \bar{z} \overline{H_{\Phi} f}$ is a maximizing vector of $H_{\Phi^{\mathrm{t}}}$. The functions $f$ and $g$ admit factorizations

$$
f=\vartheta_{1} h v, \quad g=\vartheta_{2} h w,
$$

where $h$ is a scalar outer function, $\vartheta_{1}$ and $\vartheta_{2}$ are scalar inner functions, and $v$ and $w$ are inner and co-outer column functions.

By Theorem 3.1, the column functions $v$ and $w$ have thematic (1-balanced) completions:

$$
V=(v \bar{\Theta}) \text { and } W^{\mathrm{t}}=(w \bar{\Xi}) .
$$

The function $\Phi-F$ admits the following factorization:

$$
\Phi-F=W^{*}\left(\begin{array}{cc}
t_{0} u & \mathbf{0} \\
\mathbf{0} & \Psi
\end{array}\right) V^{*},
$$

where $u=\bar{z} \bar{\vartheta}_{1} \bar{\vartheta}_{2} \bar{h} / h$ and $\|\Psi\|_{L^{\infty}} \leq t_{0}=t_{0}(\Phi)=\left\|H_{\Phi}\right\|$ (see [Pe2, Chapter 14, $\left.\S 18\right]$ ). Moreover, under the assumption (4.1), $T_{u}$ is Fredholm and ind $T_{u}>0$.

Such factorizations are called partial thematic factorizations of order 1.

As in the case of finite matrix functions (see [PT2] or [Pe2, Chapter 14, $\S 4]$ ), the following crucial inequality holds:

$$
\left\|H_{\Psi}\right\|_{\mathrm{e}} \leq\left\|H_{\Phi}\right\|_{\mathrm{e}}
$$


Another important result that can be established in the same way as in the case of finite matrix functions is that, under the assumption (4.1), the operator functions $\Theta$ and $\Xi$ are left-invertible in $H^{\infty}$ (see [PT2] or [Pe2, Chapter 14, §4]).

A function $\Phi$ satisfying (4.1) is badly approximable if and only if it admits a partial thematic factorization of order 1 :

$$
\Phi=W^{*}\left(\begin{array}{cc}
t_{0} u & \mathbf{0} \\
\mathbf{0} & \Psi
\end{array}\right) V^{*}
$$

(the "if" part is true even without the assumption (4.1)). Moreover, $\Phi$ is very badly approximable if and only if $\Psi$ is very badly approximable.

If $\Phi$ is admissible and $H_{\Psi} \neq 0$, then, due to inequality (4.2), we can apply the same procedure to $\Psi$. If $F \in \boldsymbol{\Omega}_{1}$, then $\Phi-F$ admits a thematic factorization of order 2, i.e.,

$$
\Phi-F=W^{*}\left(\begin{array}{cc}
1 & \mathbf{0} \\
\mathbf{0} & W_{1}^{*}
\end{array}\right)\left(\begin{array}{ccc}
t_{0} u_{0} & \mathbf{0} & \mathbf{0} \\
\mathbf{0} & t_{1} u_{1} & \mathbf{0} \\
\mathbf{0} & \mathbf{0} & \Lambda
\end{array}\right)\left(\begin{array}{cc}
1 & \mathbf{0} \\
\mathbf{0} & V_{1}^{*}
\end{array}\right) V^{*},
$$

where $V, V_{1}, W^{\mathrm{t}}, W_{1}^{\mathrm{t}}$ are thematic operator functions, $u_{0}$ and $u_{1}$ are scalar very badly approximable functions such that $\left\|H_{u_{j}}\right\|_{\mathrm{e}}<1$, and $\|\Lambda\|_{L^{\infty}} \leq t_{1}$.

If $\Phi$ is admissible, we can continue this process and obtain a partial thematic factorization of an arbitrary order.

In particular, an admissible operator function $\Phi$ is very badly approximable if and only if for each positive integer $r$ it admits a partial thematic factorization of order $r$.

\section{§5. Partial canonical factorizations}

As in the case of finite matrix functions (see PT3]), to obtain a geometric characterization of very badly approximable operator functions, it is more important to deal with canonical factorizations rather than with thematic factorizations.

Theorem 5.1. Let $\Phi$ be a matrix function in $L^{\infty}\left(\mathcal{B}\left(\ell^{2}\right)\right)$ such that $\left\|H_{\Phi}\right\|_{\mathrm{e}}<\left\|H_{\Phi}\right\|$, and let $r$ be the multiplicity of the superoptimal singular value $t_{0}(\Phi)$. Suppose that $\mathcal{M}$ is the minimal shift-invariant subspace of $H^{2}\left(\ell^{2}\right)$ that contains all maximizing vectors of $H_{\Phi}$. Then $\mathcal{M}=\Upsilon H^{2}\left(\mathbb{C}^{r}\right)$, where $\Upsilon$ is an inner and co-outer function of size $\infty \times r$.

Proof. Since $\mathcal{M}$ is shift-invariant, it has the form $\mathcal{M}=\Upsilon H^{2}(\mathcal{K})$, where $\mathcal{K}$ is a separable Hilbert space and $\Upsilon$ is an inner operator function. Since $\left\|H_{\Phi}\right\|_{\mathrm{e}}<\left\|H_{\Phi}\right\|$, it is easily seen that the space of maximizing vectors of $H_{\Phi}$ is finite-dimensional, and so $\operatorname{dim} \mathcal{K}<\infty$. Put $d=\operatorname{dim} \mathcal{K}$ and $\mathcal{K}=\mathbb{C}^{d}$.

We show that $d \geq r$. In [PY1] (see also Lemma 1.2 of [PY2]), in the case of finite matrix functions of class $H^{\infty}+C$ a finite sequence

$$
f_{1}^{(0)}, \ldots, f_{k_{0}}^{(0)}, f_{1}^{(1)}, \ldots, f_{k_{1}}^{(1)}, \ldots, f_{1}^{(r-1)}, \ldots, f_{k_{r-1}}^{(r-1)}
$$

of maximizing vectors of $H_{\Phi}$ was constructed. It is easy to verify that it has the following property:

$$
\max _{\zeta \in \mathbb{D}} \operatorname{dim} \operatorname{span}\left\{f_{k}^{(j)}(\zeta): 0 \leq j \leq r-1,1 \leq k \leq k_{j}\right\}=r .
$$

This construction was generalized in PT1] to the case of finite matrix functions $\Phi$ satisfying the condition $\left\|H_{\Phi}\right\|_{\mathrm{e}}<\left\|H_{\Phi}\right\|$ and in [PT2] to the case of infinite matrix functions $\Phi$ such that $H_{\Phi}$ is compact (see also Chapter 14 of [Pe2]). It can easily be verified that exactly the same construction also works in the case of infinite matrix functions $\Phi$ satisfying the condition $\left\|H_{\Phi}\right\|_{\mathrm{e}}<\left\|H_{\Phi}\right\|$ and (5.1) holds. From (5.1), it follows immediately that $d \geq r$. 
Let us now show that $d \leq r$. Let $F$ be a function in $\boldsymbol{\Omega}_{r}$. Consider a partial canonical factorization of $\Phi-F$. It has the form

$$
\Phi-F=\mathfrak{W}\left(\begin{array}{cc}
t_{0} \mathfrak{U} & \mathbf{0} \\
\mathbf{0} & \Psi
\end{array}\right) \mathfrak{V}
$$

where $\mathfrak{W}$ and $\mathfrak{V}$ are infinite unitary-valued functions, $\mathfrak{U}$ is an $r \times r$ unitary-valued function, and $\|\Psi\|_{L^{\infty}}=t_{r}<t_{0}$. It follows that the subspace spanned by the maximizing vectors of $(\Phi-F)(\zeta)$ has dimension $r$ for almost all $\zeta \in \mathbb{T}$.

For every function $f \in \mathcal{M}$ the vector $f(\zeta)$ is a maximizing vector of $\Phi(\zeta)$ for almost all $\zeta \in \mathbb{T}$ (see Lemma 15.2 in Chapter 14 of [Pe2]; note that in $[\mathrm{Pe} 2]$ the result is stated for finite matrix functions, but the proof given there works for infinite matrix functions too). Now it is easy to check that if $d>r$, then the subspace spanned by the maximizing vectors of $(\Phi-F)(\zeta)$ has dimension at least $d$.

It remains to show that $\Upsilon$ is co-outer. Without loss of generality we may assume that $\|\Phi\|_{\infty}=\left\|H_{\Phi}\right\|=1$. The subspace of $H^{2}\left(\ell^{2}\right)$ spanned by the maximizing vectors of $H_{\Phi}$ must be finite-dimensional. Let $f_{1}, \ldots, f_{s}$ be a basis of this subspace, and let $F$ be the matrix function whose columns are $f_{1}, \ldots, f_{s}$. Consider the inner-outer factorization of $F^{\mathrm{t}}$ :

$$
F^{\mathrm{t}}=\mathcal{O} G
$$

where $\mathcal{O}$ is an inner matrix function of size $s \times k, k \leq s$, and $G$ is an outer matrix function of size $k \times \infty$. Then

$$
F=G^{\mathrm{t}} \mathcal{O}^{\mathrm{t}},
$$

whence

$$
G^{\mathrm{t}}=G^{\mathrm{t}} \mathcal{O}^{\mathrm{t}} \overline{\mathcal{O}}=F \overline{\mathcal{O}}
$$

Since the functions $f_{j}$ are maximizing vectors of $H_{\Phi}$, it follows that for almost all $\zeta \in \mathbb{T}$ the vectors $f_{j}(\zeta)$ are maximizing vectors of $\Phi(\zeta)$ and $\Phi f_{j} \in H_{-}^{2}\left(\ell^{2}\right)$ (see Pe2, Chapter 2 , Theorem 2.3]). Thus for almost all $\zeta \in \mathbb{T}$, the restriction of $\Phi(\zeta)$ to Range $F(\zeta)$ is an isometry and $\Phi F \in H_{-}^{2}\left(\mathcal{B}\left(\mathbb{C}^{s}, \ell^{2}\right)\right)$. Consequently,

$$
\Phi G^{\mathrm{t}}=\Phi F \overline{\mathcal{O}} \in H_{-}^{2}\left(\mathcal{B}\left(\mathbb{C}^{k}, \ell^{2}\right)\right) .
$$

Suppose now that $g$ is a column of $G^{\mathrm{t}}$. Then $\Phi g \in H_{-}^{2}\left(\ell^{2}\right)$. Since

$$
g(\zeta) \in \text { Range } G^{\mathrm{t}}(\zeta) \subset \text { Range } F(\zeta) \quad \text { for almost all } \zeta \in \mathbb{T} \text {, }
$$

it follows that

$$
\|\Phi(\zeta) g(\zeta)\|_{\ell^{2}}=\|g(\zeta)\|_{\ell^{2}} \quad \text { almost everywhere on } \mathbb{T} \text {. }
$$

Thus

$$
\left\|H_{\Phi} g\right\|=\left\|\mathbb{P}_{-} \Phi g\right\|=\|\Phi g\|=\|g\|,
$$

and so all columns of $G^{\mathrm{t}}$ are maximizing vectors of $H_{\Phi}$. Since the columns of $F$ form a basis in the space of maximizing vectors, formula (5.2) implies that $\mathcal{O}$ is a constant isometric matrix.

Clearly, $\mathcal{M}$ is the minimal invariant subspace of multiplication by $z$ on $H^{2}\left(\ell^{2}\right)$ that contains the columns of $F$. Consider the minimal invariant subspace $\mathcal{M}_{1}$ that contains the columns of $G^{\mathrm{t}}$. Since $F=G^{\mathrm{t}} \mathcal{O}^{\mathrm{t}}$ and $\mathcal{O}$ is a constant matrix, we have $\mathcal{M} \subset \mathcal{M}_{1}$. On the other hand, the columns of $G^{\text {t }}$ are maximizing vectors of $H_{\Phi}$, and so $\mathcal{M}_{1} \subset \mathcal{M}$. Thus $\mathcal{M}_{1}=\mathcal{M}$.

Now it is easy to see that $\Upsilon$ is the inner factor of the inner-outer factorization of $G^{\mathrm{t}}$. It follows now from Theorem 2.1 that $\Upsilon$ is co-outer. 
Consider now the matrix function $\Phi^{\mathrm{t}}$. Let $\mathcal{N}$ be the shift-invariant subspace of $H^{2}\left(\ell^{2}\right)$ spanned by the maximizing vectors of $H_{\Phi^{\mathrm{t}}}$. By Theorem 5.1 $\mathcal{N}$ has the form $\Omega H^{2}\left(\mathbb{C}^{r}\right)$, where $\Omega$ is an inner and co-outer matrix function. By Theorem 3.1, there exist inner and co-outer matrix functions $\Theta$ and $\Xi$ such that

$$
\mathcal{V} \stackrel{\text { def }}{=}(\Upsilon \bar{\Theta}) \text { and } \quad \mathcal{W}^{\text {t }} \stackrel{\text { def }}{=}(\Omega \bar{\Xi})
$$

are unitary-valued matrix functions.

The proof of the following result is exactly the same as the proof of Theorem 15.3 in Chapter 14 of $\mathrm{Pe} 2$ for finite matrix functions (see also $\mathrm{AP}$ ).

Theorem 5.2. Let $\Phi$ be a function in $L^{\infty}\left(\mathcal{B}\left(\ell^{2}\right)\right)$ such that $\left\|H_{\Phi}\right\|_{\mathrm{e}}<t_{0}=\left\|H_{\Phi}\right\|$. Let $r$ be the number of superoptimal singular values of $\Phi$ equal to $t_{0}$. Suppose that $F$ is a best approximant for $\Phi$ by analytic matrix functions. Then $\Phi-F$ admits a factorization of the form

$$
\Phi-F=\mathcal{W}^{*}\left(\begin{array}{cc}
t_{0} U & \mathbf{0} \\
\mathbf{0} & \Psi
\end{array}\right) \mathcal{V}^{*},
$$

where $\mathcal{V}$ and $\mathcal{W}$ are given by (5.3), $U$ is an $r \times r$ unitary-valued very badly approximable matrix function such that $\left\|H_{U}\right\|_{\mathrm{e}}<1$, and $\Psi$ is a matrix function in $L^{\infty}\left(\mathcal{B}\left(\ell^{2}\right)\right)$ such that $\|\Psi\|_{L^{\infty}} \leq t_{0}$ and $\left\|H_{\Psi}\right\|=t_{r}(\Phi)<\left\|H_{\Phi}\right\|$. Moreover, $U$ is uniquely determined by the choice of $\Upsilon$ and $\Omega$ and does not depend on the choice of $F$.

As in the case of finite matrix functions, under the hypotheses of Theorem 5.2 the following inequality holds:

$$
\left\|H_{\Psi}\right\|_{\mathrm{e}} \leq\left\|H_{\Phi}\right\|_{\mathrm{e}} .
$$

This can be deduced from (4.2) in exactly the same way as in AP (see also Theorem 15.12 of Chapter 14 of [Pe2]).

Moreover, under the hypotheses of Theorem [5.2, the operator functions $\Theta$ and $\Xi$ in (5.3) are left-invertible in $H^{\infty}$. Again, this can be deduced from the same results for partial thematic factorizations (see $\$ 4$ ) in the same way as this was done in the case of finite matrix functions in $\mathrm{AP}$ (see also $\underline{\mathrm{Pe} 2}$, Chapter 14, §5]). This left-invertibility property of $\Theta$ and $\Xi$ is important in the main result of the next section.

The following theorem can be viewed as a converse to Theorem 5.2. These two theorems together give a characterization of the badly approximable matrix functions $\Phi$ satisfying the condition $\left\|H_{\Phi}\right\|_{\mathrm{e}}<t_{0}=\left\|H_{\Phi}\right\|$. Note however that we do not need this condition to prove that the functions that admit a factorization of the form (5.4) are badly approximable. Moreover, in the following theorem we can also relax the assumptions on $U$ imposed in Theorem 5.2.

Theorem 5.3. Let $\Phi$ be an infinite matrix function of the form

$$
\Phi=\mathcal{W}^{*}\left(\begin{array}{cc}
\sigma U & 0 \\
0 & \Psi
\end{array}\right) \mathcal{V}^{*}
$$

where $\sigma>0, \mathcal{V}$ and $\mathcal{W}^{\mathrm{t}}$ are $r$-balanced matrix functions, $U$ is an $r \times r$ unitary-valued matrix function such that the shift-invariant subspace of $H^{2}\left(\mathbb{C}^{r}\right)$ spanned by the maximizing vectors of $H_{U}$ coincides with $H^{2}\left(\mathbb{C}^{r}\right)$, and $\|\Psi\|_{\infty} \leq \sigma$. Then $\Phi$ is badly approximable and $t_{0}(\Phi)=\cdots=t_{r-1}(\Phi)=\sigma$. Moreover, $\Phi$ is very badly approximable if and only if $\Psi$ is very badly approximable.

The proof of Theorem 5.3 is exactly the same as the proof of Theorem 15.7 of Chapter 14 of $\mathrm{Pe} 2$ for finite matrix functions (see also $\mathrm{AP}$ ).

Now, consider the sequence

$$
t_{0}=\cdots=t_{r_{1}-1}>t_{r_{1}}=\cdots=t_{r_{2}-1}>\cdots>t_{r_{\iota-1}}=\cdots=t_{r_{\iota}-1}>\cdots
$$


of superoptimal singular values of $\Phi$. Let $\sigma_{0}>\sigma_{1}>\sigma_{2}>\cdots$ be the sequence of distinct superoptimal singular values of $\Phi$, i.e.,

$$
\sigma_{0}=t_{0}=\cdots=t_{r_{1}-1}, \quad \sigma_{1}=t_{r_{1}}=\cdots=t_{r_{2}-1}, \quad \text { etc. }
$$

If $\left\|H_{\Phi}\right\|_{\mathrm{e}}<\sigma_{1}$, we can apply Theorem 5.2 to the matrix function $\Psi$. Now if $\left\|H_{\Phi}\right\|_{\mathrm{e}}<$ $\sigma_{2}$, then $\left\|H_{\Psi}\right\|_{\mathrm{e}}<\sigma_{2}$ by (5.5), and so we can continue this process and obtain the following result in exactly the same way as in the case of finite matrix functions in $\mathrm{Pe} 2$, Chapter 14, §15].

Theorem 5.4. Let $\Phi$ be a function in $L^{\infty}\left(\mathcal{B}\left(\ell^{2}\right)\right)$ such that $\left\|H_{\Phi}\right\|_{\mathrm{e}}<\sigma_{d-1}$. Let $F$ be an arbitrary matrix function in $\boldsymbol{\Omega}_{r_{d}}$. Then $\Phi-F$ admits a factorization

$$
\Phi-F=\mathcal{W}_{0}^{*} \cdots \mathcal{W}_{d-1}^{*}\left(\begin{array}{ccccc}
\sigma_{0} U_{0} & \mathbf{0} & \cdots & \mathbf{0} & \mathbf{0} \\
\mathbf{0} & \sigma_{1} U_{1} & \cdots & \mathbf{0} & \mathbf{0} \\
\vdots & \vdots & \ddots & \vdots & \vdots \\
\mathbf{0} & \mathbf{0} & \cdots & \sigma_{d-1} U_{d-1} & \mathbf{0} \\
\mathbf{0} & \mathbf{0} & \cdots & \mathbf{0} & \Psi
\end{array}\right) \mathcal{V}_{d-1}^{*} \cdots \mathcal{V}_{0}^{*},
$$

where the $U_{j}$ are $\left(r_{j+1}-r_{j}\right) \times\left(r_{j+1}-r_{j}\right)$ very badly approximable unitary-valued functions such that $\left\|H_{U_{j}}\right\|_{e}<1$,

$$
\mathcal{V}_{j}=\left(\begin{array}{cc}
\boldsymbol{I}_{r_{j}} & \mathbf{0} \\
\mathbf{0} & \breve{\mathcal{V}}_{j}
\end{array}\right) \text { and } \mathcal{W}_{j}=\left(\begin{array}{cc}
\boldsymbol{I}_{r_{j}} & \mathbf{0} \\
\mathbf{0} & \breve{\mathcal{W}}_{j}
\end{array}\right), \quad 1 \leq j \leq d-1,
$$

the $\breve{\mathcal{V}}_{j}$ and $\breve{\mathcal{W}}_{j}^{\mathrm{t}}$ are $\left(r_{j+1}-r_{j}\right)$-balanced matrix functions, and $\Psi$ is a matrix function satisfying

$$
\|\Psi\|_{L^{\infty}} \leq t_{r_{d-1}} \text { and } \quad\left\|H_{\Psi}\right\|<t_{r_{d-1}} .
$$

Factorizations of the form (5.7) with the $\sigma_{j}, U_{j}, \mathcal{V}_{j}$, and $\mathcal{W}_{j}$ as in Theorem 5.4 are called partial canonical factorizations (or partial canonical factorizations of order $d$ ).

Now we can state the following description of the very badly approximable matrix functions.

Theorem 5.5. Let $\Phi$ be an admissible function in the space $L^{\infty}\left(\mathcal{B}\left(\ell^{2}\right)\right)$. If $\Phi$ is very badly approximable, then for each $d$ with nonzero $\sigma_{d-1}$ the matrix function $\Phi$ admits a partial canonical factorization of the form (5.7).

The proof of Theorem [5.5] is exactly the same as in the case of finite matrix functions treated in [Pe2, Chapter 14, §15] (see also [AP]). Finally, we state the converse to Theorem [5.5] which is valid without the admissibility assumption.

Theorem 5.6. Let $\Phi$ be a function in $L^{\infty}\left(\mathcal{B}\left(\ell^{2}\right)\right)$ such that $\Phi$ admits a partial canonical factorization of the form (5.7) whenever $\sigma_{d-1}>0$. Then $\Phi$ is very badly approximable and

$$
t_{\varkappa}(\Phi)= \begin{cases}\sigma_{0}, & \varkappa<r_{1}, \\ \sigma_{j}, & r_{j} \leq \varkappa<r_{j+1} .\end{cases}
$$

Example. As was mentioned in the Introduction, there is an important difference between the case of finite matrix functions and the case of infinite matrix functions. In the case of finite matrix functions the hypotheses of Theorem 5.5 guarantee that the zero function is the only superoptimal approximant. It turns out that in the case of infinite matrix functions this is not true. Consider the following example.

Let $\left\{u_{j}\right\}_{j \geq 0}$ be a sequence of scalar badly approximable functions such that

$$
\left|u_{j}(\zeta)\right|=1 \text { for almost all } \zeta \in \mathbb{T} \text { and }\left\|H_{u_{j}}\right\|_{\mathrm{e}}<1,
$$


and let $\left\{t_{j}\right\}_{j \geq 0}$ be a decreasing sequence of positive numbers such that

$$
\lim _{j \rightarrow \infty} t_{j}>0 .
$$

Consider the infinite matrix function

$$
\Phi=\left(\begin{array}{ccccc}
\mathbf{0} & \mathbf{0} & \mathbf{0} & \mathbf{0} & \cdots \\
\mathbf{0} & t_{0} u_{0} & \mathbf{0} & \mathbf{0} & \cdots \\
\mathbf{0} & \mathbf{0} & t_{1} u_{1} & \mathbf{0} & \cdots \\
\mathbf{0} & \mathbf{0} & \mathbf{0} & t_{2} u_{2} & \cdots \\
\vdots & \vdots & \vdots & \vdots & \ddots
\end{array}\right) .
$$

Obviously, for every $d \in \mathbb{Z}_{+}$there is a constant unitary matrix $V_{d}$ such that

$$
\Phi=V_{d}^{*}\left(\begin{array}{ccccccc}
t_{0} u_{0} & \mathbf{0} & \mathbf{0} & \cdots & \mathbf{0} & \mathbf{0} & \cdots \\
\mathbf{0} & t_{1} u_{1} & \mathbf{0} & \cdots & \mathbf{0} & \mathbf{0} & \cdots \\
\vdots & \vdots & \ddots & \vdots & \vdots & \vdots & \vdots \\
\mathbf{0} & \mathbf{0} & \cdots & t_{d-1} u_{d-1} & \mathbf{0} & \mathbf{0} & \cdots \\
\mathbf{0} & \mathbf{0} & \cdots & \mathbf{0} & \mathbf{0} & \mathbf{0} & \cdots \\
\mathbf{0} & \mathbf{0} & \cdots & \mathbf{0} & \mathbf{0} & t_{d} u_{d} & \cdots \\
\vdots & \vdots & \vdots & \vdots & \vdots & \vdots & \ddots
\end{array}\right) V_{d} .
$$

Clearly, the right-hand side of (5.8) is a partial canonical factorization of $\Phi$, and Theorem 5.6 shows that $\Phi$ is very badly approximable and $t_{j}(\Phi)=t_{j}$.

On the other hand, if $f$ is an arbitrary scalar function in $H^{\infty}$ with

$$
\|f\|_{\infty} \leq \lim _{j \rightarrow \infty} t_{j}
$$

and

$$
F=\left(\begin{array}{cccc}
f & \mathbf{0} & \mathbf{0} & \cdots \\
\mathbf{0} & \mathbf{0} & \mathbf{0} & \cdots \\
\mathbf{0} & \mathbf{0} & \mathbf{0} & \cdots \\
\vdots & \vdots & \vdots & \ddots
\end{array}\right)
$$

then

$$
\Phi-F=\left(\begin{array}{ccccc}
-f & \mathbf{0} & \mathbf{0} & \mathbf{0} & \cdots \\
\mathbf{0} & t_{0} u_{0} & \mathbf{0} & \mathbf{0} & \cdots \\
\mathbf{0} & \mathbf{0} & t_{1} u_{1} & \mathbf{0} & \cdots \\
\mathbf{0} & \mathbf{0} & \mathbf{0} & t_{2} u_{2} & \cdots \\
\vdots & \vdots & \vdots & \vdots & \ddots
\end{array}\right),
$$

and since, obviously,

$$
s_{j}((\Phi-F)(\zeta))=t_{j}, \quad j \in \mathbb{Z}_{+}, \quad \zeta \in \mathbb{T},
$$

it follows that $F$ is a superoptimal approximant of $\Phi$.

To make the conclusion that an admissible infinite matrix function has a unique superoptimal approximant, we need the condition that

$$
\lim _{j \rightarrow \infty} t_{j}(\Phi)=0 .
$$

Indeed, if $F_{1}$ and $F_{2}$ belong to $\boldsymbol{\Omega}_{r}$, then we can consider partial thematic factorizations of $\Phi-F_{1}$ and $\Phi-F_{2}$ to see that

$$
\left\|F_{1}-F_{2}\right\|_{\infty}=\left\|\left(\Phi-F_{1}\right)-\left(\Phi-F_{2}\right)\right\|_{\infty} \leq 2 t_{r}(\Phi) .
$$


In particular, if both $F_{1}$ and $F_{2}$ are superoptimal approximants, then $F_{1}=F_{2}$ by (5.9). However, if $\Phi$ is admissible and satisfies (5.9), then $\lim _{j \rightarrow \infty} s_{j}\left(H_{\Phi}\right)=0$, and so $H_{\Phi}$ is compact.

\section{§6. VERY BADLY APPROXIMABLE FUNCTIONS}

In this section we obtain a necessary and sufficient condition for an admissible infinite matrix function to be very badly approximable. Let $\Phi \in L^{\infty}\left(\mathcal{B}\left(\ell^{2}\right)\right)$. Put

$$
t_{\infty}(\Phi)=\lim _{j \rightarrow \infty} t_{j}(\Phi) .
$$

As in the case of finite matrix functions, for $\sigma>t_{\infty}(\Phi)$ we consider the subspace $\mathfrak{S}_{\Phi}^{(\sigma)}(\zeta)$, which is the linear span of the Schmidt vectors of $\Phi(\zeta)$ that correspond to the

singular values of $\Phi(\zeta)$ that are greater than or equal to $\sigma$. The subspaces $\mathfrak{S}_{\Phi}^{(\sigma)}(\zeta)$ are defined for almost all $\zeta \in \mathbb{T}$.

Definition. Let $L(\zeta), \zeta \in \mathbb{T}$, be a family of subspaces of $\ell^{2}$ that is defined almost everywhere on $\mathbb{T}$. We say that functions $\xi_{1}, \ldots, \xi_{l}$ in $H^{2}\left(\ell^{2}\right)$ span the family $L$ if $L(\zeta)=$ $\operatorname{span}\left\{\xi_{j}(\zeta): 1 \leq j \leq l\right\}$ for almost all $\zeta \in \mathbb{T}$.

In this section, we consider the following condition:

(C) for each $\sigma>t_{\infty}(\Phi)$, the family of subspaces $\mathfrak{S}_{\Phi}^{(\sigma)}$ is analytic and spanned by finitely many functions in $\operatorname{Ker} T_{\Phi}$.

As in the case of finite matrix functions (see PT3), it is easy to show that condition (C) implies that the functions $\zeta \mapsto s_{j}(\Phi(\zeta)), j \in \mathbb{Z}_{+}$, are constant almost everywhere on $\mathbb{T}$.

The following theorem is the main result of this section.

Theorem 6.1. If $\Phi$ is an admissible very badly approximable matrix function in the space $L^{\infty}\left(\mathcal{B}\left(\ell^{2}\right)\right)$, then $\Phi$ satisfies $(\mathrm{C})$.

Conversely, if $\Phi$ is an arbitrary function in $L^{\infty}\left(\mathcal{B}\left(\ell^{2}\right)\right)$ that satisfies $(\mathrm{C})$, then $\Phi$ is very badly approximable.

Remark. As we have already mentioned, there is an important difference between the case of finite matrix functions and the case of infinite matrix functions. In the case of finite matrix functions condition $(\mathrm{C})$ also implies that the zero function is the only superoptimal approximant. In the case of infinite matrix functions this is not true. Indeed, it is easy to see that the matrix function given in the example at the end of the preceding section satisfies condition (C). However, it has infinitely many superoptimal approximants.

The necessity of condition (C) can be obtained from Theorem 5.5 in exactly the same way as was done in [PT3, Theorem 4.1] in the case of finite matrix functions. On the other hand, the proof of the sufficiency of (C) given in [PT3] works only for finite matrix functions. It must be modified slightly to work in the case of infinite matrix functions.

Here we present a proof based on canonical factorizations. Note that the proof based on superoptimal weights that was presented in $\S 5$ of [PT3 in the case of finite matrix functions, also works (with obvious modifications).

Proof of the sufficiency of $(\mathrm{C})$. Suppose that $\Phi$ satisfies $(\mathrm{C})$. As we have already observed, the functions $\zeta \mapsto s_{j}(\Phi(\zeta)), j \in \mathbb{Z}_{+}$, are constant almost everywhere on $\mathbb{T}$. Let

$$
\sigma_{0}>\sigma_{1}>\sigma_{2}>\cdots
$$


be positive numbers (finitely many or infinitely many) such that for almost all $\zeta \in \mathbb{T}$, the numbers (6.1) are all nonzero distinct singular values of $\Phi(\zeta)$. It suffices to prove that if $\sigma_{d-1}>0$, then $\Phi$ admits a partial canonical factorization of order $d$. We prove this by induction on $d$.

First, suppose that $d=1$. Let $r=\operatorname{dim} \mathfrak{S}_{\Phi}^{\left(\sigma_{0}\right)}(\zeta)$ for almost all $\zeta \in \mathbb{T}$. Obviously, $\operatorname{dim} \mathfrak{S}_{\Phi^{t}}^{\left(\sigma_{0}\right)}(\zeta)=r$ for almost all $\zeta \in \mathbb{T}$. We show that $\Phi$ admits a factorization of the form (5.6) with $r$-balanced functions $\mathcal{V}$ and $\mathcal{W}^{\mathrm{t}}$. It is easy to verify that a function $\xi \in H^{2}\left(\ell^{2}\right)$ is a maximizing vector of $H_{\Phi}$ if and only if $\eta \stackrel{\text { def }}{=} \bar{z} \overline{H_{\Phi} \xi}$ is a maximizing vector of $H_{\Phi^{t}}$ (see [Pe2, Chapter 14, §2]). Let $\mathcal{M}$ be the minimal invariant subspace of multiplication by $z$ on $H^{2}\left(\ell^{2}\right)$ that contains all maximizing vectors of $H_{\Phi}$, and let $\mathcal{N}$ be the minimal invariant subspace of multiplication by $z$ on $H^{2}\left(\ell^{2}\right)$ that contains all maximizing vectors of $H_{\Phi^{\mathrm{t}}}$.

By Theorem 5.1, there exist inner and co-outer functions $\Upsilon$ and $\Omega$ in $H^{\infty}\left(\mathcal{B}\left(\mathbb{C}^{r}, \ell^{2}\right)\right)$ such that $\mathcal{M}=\Upsilon H^{2}\left(\mathbb{C}^{r}\right)$ and $\mathcal{N}=\boldsymbol{O} H^{2}\left(\mathbb{C}^{r}\right)$. By Theorem 3.1, there exist $r$-balanced matrix functions $\mathcal{V}$ and $\mathcal{W}^{\mathrm{t}}$ of the form

$$
\mathcal{V}=(\Upsilon \bar{\Theta}) \text { and } \mathcal{W}^{\mathrm{t}}=(\Omega \bar{\Xi}) \text {. }
$$

In exactly the same way as in the proof of Theorem 3.2 in [PT3], it can be shown that $\Phi$ admits a factorization

$$
\Phi=\mathcal{W}^{*}\left(\begin{array}{cc}
\sigma_{0} U & \mathbf{0} \\
\mathbf{0} & \Psi
\end{array}\right) \mathcal{V}^{*},
$$

where $U$ is an $r \times r$ unitary-valued matrix function. The fact that the shift-invariant subspace spanned by the maximizing vectors of $H_{U}$ is $H^{2}\left(\mathbb{C}^{r}\right)$ is verified in the same way as was done in the proof of Theorem 4.1 in PT3.

In exactly the same way as in the proof of Theorem 4.1 in [PT3], we can show that $\Psi$ satisfies condition (C). Clearly, for almost all $\zeta \in \mathbb{T}, \sigma_{1}>\sigma_{2}>\cdots$ are all nonzero distinct singular values of $\Psi(\zeta)$.

Suppose now that $d>1$. By the inductive hypothesis, $\Psi$ admits a partial canonical factorization of order $d-1$. Thus, $\Phi$ admits a partial canonical factorization of order $d$.

Remarks on uniqueness. As was mentioned above, unlike the case of finite matrix functions, in the infinite-dimensional case a very badly approximable function satisfying condition (C) can have infinitely many superoptimal approximants. However, in certain important cases the zero function is the only superoptimal approximant of a very badly approximable function $\Phi$ :

(i) if the Hankel operator $H_{\Phi}$ is compact, then $\Phi$ has a unique superoptimal approximant (see [T, Pe1, PT1]), and so in this case (note that such functions $\Phi$ are automatically admissible) $\Phi$ is very badly approximable if and only if condition (C) is fulfilled, and in this case the zero function is the only superoptimal approximant of $\Phi$;

(ii) if $\operatorname{rank} \Phi(\zeta)$ is uniformly bounded for almost all $\zeta \in \mathbb{T}$ (this happens, for example, if $\Phi$ has finitely many columns or rows), then the family of subspaces $\mathfrak{S}_{\Phi}^{(\sigma)}$ stabilizes and we have the situation similar to the case of finite matrix functions; in this case, again, the zero function is the only superoptimal approximant of $\Phi$ provided $\Phi$ satisfies condition $(\mathrm{C})$;

(iii) if $\Phi$ satisfies condition (C) and for almost all $\zeta \in \mathbb{T}$ the subspaces $\mathfrak{S}_{\Phi}^{(\sigma)}(\zeta)$, $\sigma>t_{\infty}(\Phi)$, span $\ell^{2}$, then it is not hard to see that the zero function is the only superoptimal approximant of $\Phi$.

We explain (iii) in more detail. 
Suppose $\Phi$ satisfies condition (C), and let $\sigma_{k}, k \geq 0$, be the decreasing sequence such that for almost all $\zeta \in \mathbb{T}$, the $\sigma_{k}$ are all nonzero distinct singular values of $\Phi(\zeta)$ (we have already mentioned that $(\mathrm{C})$ implies that the singular values of $\Phi(\zeta)$ are constant for almost all $\zeta \in \mathbb{T})$.

In $\S 5$ of [PT3] it was shown that if $F$ is a superoptimal approximation of $\Phi$, then

$$
(\Phi(\zeta)-F(\zeta))\left|\mathfrak{S}_{\Phi}^{\left(\sigma_{k}\right)}(\zeta)=\Phi(\zeta)\right| \mathfrak{S}_{\Phi}^{\left(\sigma_{k}\right)}(\zeta) \quad \text { for almost all } \zeta \in \mathbb{T}
$$

(this was done in PT3 for finite matrix functions, but the same proof also works in the infinite-dimensional case). Thus if we assume that the subspaces $\mathfrak{S}_{\Phi}^{\left(\sigma_{k}\right)}(\zeta)$ span $\ell^{2}$ for almost all $\zeta \in \mathbb{T}$, we obtain $\Phi-F=\Phi$, and so the zero function is the only superoptimal approximant of $\Phi$.

\section{§7. BADLY APPROXIMABLE OPERATOR FUNCTIONS}

In PT3 we obtained a description of the badly approximable matrix functions. Now we can obtain the same result for operator functions.

Theorem 7.1. Let $\Phi \in L^{\infty}\left(\mathcal{B}\left(\ell^{2}\right)\right)$, and let $\left\|H_{\Phi}\right\|_{\mathrm{e}}<\|\Phi\|_{L^{\infty}}$. If $\Phi$ is badly approximable, then

(i) $\|\Phi(\zeta)\|_{\mathcal{B}\left(\ell^{2}\right)}$ is constant for almost all $\zeta \in \mathbb{T}$;

(ii) there exists a function $f$ in $\operatorname{Ker} T_{\Phi}$ such that $f(\zeta)$ is a maximizing vector of $\Phi(\zeta)$ for almost all $\zeta \in \mathbb{T}$.

Conversely, if $\Phi$ belongs to $L^{\infty}\left(\mathcal{B}\left(\ell^{2}\right)\right)$ and satisfies (i) and (ii), then $\Phi$ is badly approximable.

The arguments are exactly the same as in the proof of Theorem 6.1 of PT3].

Another result of $\S 6$ of [PT3] is a characterization of the set of badly approximable functions $\Phi$ such that $\left\|H_{\Phi}\right\|_{\mathrm{e}}<\|\Phi\|_{L^{\infty}}$ and $\mathbf{0}$ is the only best approximant of $\Phi$. We can ask the same question in the case of infinite matrix functions. However, if $\Phi \in L^{\infty}\left(\mathcal{B}\left(\ell^{2}\right)\right)$ and $\left\|H_{\Phi}\right\|_{\mathrm{e}}<\|\Phi\|_{L^{\infty}}$, then $\mathbf{0}$ cannot be the only best approximant. Indeed, if $\Phi$ is a badly approximable function satisfying $\left\|H_{\Phi}\right\|_{\mathrm{e}}<\|\Phi\|_{L^{\infty}}$, then by Theorem 5.3 , it admits a partial canonical factorization

$$
\Phi=\mathcal{W}^{*}\left(\begin{array}{cc}
\sigma U & \mathbf{0} \\
\mathbf{0} & \Psi
\end{array}\right) \mathcal{V}^{*}
$$

and $\left\|H_{\Psi}\right\|<\sigma$. Then there are infinitely many functions $Q$ in $H^{\infty}\left(\mathcal{B}\left(\ell^{2}\right)\right)$ such that $\|\Psi-Q\|_{L^{\infty}}<\sigma$. Now it is easy to verify (see Theorem 1.8 in Chapter 14 of [Pe2]) that

$$
\Phi-\Xi Q \Theta^{\mathrm{t}}=\mathcal{W}^{*}\left(\begin{array}{cc}
\sigma U & \mathbf{0} \\
\mathbf{0} & \Psi-Q
\end{array}\right) \mathcal{V}^{*}
$$

where $\Theta$ and $\Xi$ are as in (5.3). Thus $\Phi$ has infinitely many best approximants.

However, we still can obtain a sufficient condition for a badly approximable operator function to have a unique best approximant. Clearly, such a function $\Phi$ cannot satisfy the inequality $\left\|H_{\Phi}\right\|_{\mathrm{e}}<\|\Phi\|_{L^{\infty}}$. It is convenient to normalize $\Phi$ with the condition $\|\Phi\|_{L^{\infty}}=1$.

Theorem 7.2. Let $\Phi \in L^{\infty}\left(\mathcal{B}\left(\ell^{2}\right)\right)$ be a function such that $\Phi(\zeta)$ is an isometry for almost all $\zeta \in \mathbb{T}$ or $\Phi^{*}(\zeta)$ is an isometry for almost all $\zeta \in \mathbb{T}$. Suppose that $\left\{f(\zeta): f \in \operatorname{Ker} T_{\Phi}\right\}$ is a dense subset of $\operatorname{Ker} \Phi(\zeta)^{\perp}$ for almost all $\zeta \in \mathbb{T}$. Then $\Phi$ is very badly approximable and the zero function is the only best approximant of $\Phi$. 
Proof. Clearly, the fact that $\Phi$ is very badly approximable is an immediate consequence of Theorem 7.1. Let $F$ be a best approximant of $\Phi$, and let $\Psi=\Phi-F$. Take $f \in \operatorname{Ker} T_{\Phi}$. Suppose that $\Phi(\zeta)$ is a co-isometry for almost all $\zeta \in \mathbb{T}$. By assumption, $f(\zeta) \in \operatorname{Ker} \Phi(\zeta)^{\perp}$ for almost all $\zeta \in \mathbb{T}$. Hence,

$$
\|\Phi(\zeta) f(\zeta)\|=\|f(\zeta)\| \quad \text { for almost all } \zeta \in \mathbb{T}
$$

(in the case when $\Phi(\zeta)$ is an isometry the above identity is fulfilled automatically). Since $f \in \operatorname{Ker} T_{\Phi}$, we conclude that $H_{\Phi} f=\Phi f$, and (7.1) implies that

$$
\left\|H_{\Phi} f\right\|_{2}=\|\Phi f\|_{2}=\|f\|_{2} \text {. }
$$

Since $F \in H^{\infty}$, we have $H_{\Phi}=H_{\Psi}$. In the chain of inequalities

$$
\begin{aligned}
\|f\|_{2} & =\left\|H_{\Phi} f\right\|_{2}=\left\|H_{\Psi} f\right\|_{2} \\
& \leq\|\Psi f\|_{2} \leq\|\Psi\|_{\infty}\|f\|_{2} \leq\|f\|_{2}
\end{aligned}
$$

all inequalities are, in fact, equalities; therefore

$$
\Psi f=H_{\Psi} f=H_{\Phi} f=\Phi f .
$$

Since the set $\left\{f(\zeta): f \in \operatorname{Ker} T_{\Phi}\right\}$ is a dense subset of $\operatorname{Ker} \Phi(\zeta)^{\perp}$ for almost all $\zeta \in \mathbb{T}$, we see that

$$
\Phi(\zeta)\left|\operatorname{Ker} \Phi(\zeta)^{\perp}=\Psi(\zeta)\right| \operatorname{Ker} \Phi(\zeta)^{\perp} \quad \text { for almost all } \zeta \in \mathbb{T} .
$$

If $\Phi(\zeta)$ is an isometry for almost all $\zeta \in \mathbb{T}$, then $\operatorname{Ker} \Phi(\zeta)$ is trivial, and $\Phi=\Psi$.

If $\Phi(\zeta)$ is a co-isometry for almost all $\zeta \in \mathbb{T}$, then (7.2) implies that for almost all $\zeta \in \mathbb{T}$ we have

$$
(\Phi(\zeta) x, y)=(\Psi(\zeta) x, y), \quad x \in \operatorname{Ker} \Phi(\zeta)^{\perp}, \quad y \in \ell^{2} .
$$

Since $\|\Psi\|_{\infty} \leq 1$, it follows that $\Phi^{*}(\zeta)=\Psi^{*}(\zeta)$ for almost all $\zeta \in \mathbb{T}$.

\section{REFERENCES}

[AAK] V. M. Adamyan, D. Z. Arov, and M. G. Kreı̆n, On infinite Hankel matrices and generalized problems of Carathéodory-Fejér and F. Riesz, Funktsional. Anal. i Prilozhen. 2 (1968), no. 1, 1-19; English transl. in Funct. Anal. Appl. 2 (1968), no. 1. MR0234274 (38:2591)

[AP] R. B. Alexeev and V. V. Peller, Badly approximable matrix functions and canonical factorizations, Indiana Univ. Math. J. 49 (2000), 1247-1285. MR.1836530 (2002e:47028)

[C] M. Cambern, Analytic range functions, J. Math. Anal. Appl. 12 (1965), 413-424. MR0188821 $(32: 6253)$

[D] R. G. Douglas, Banach algebra techniques in operator theory, Pure Appl. Math., vol. 49, Acad. Press, New York-London, 1972. MR0361893 (50:14335)

$[\mathrm{H}] \quad$ H. Helson, Lectures on invariant subspaces, Acad. Press, New York-London, 1964. MR0171178 (30:1409)

[HL] H. Helson and D. Lowdenslager, Prediction theory and Fourier series in several variables. II, Acta Math. 106 (1961), 175-213. MR.0176287 (31:562)

[Kh] S. Ya. Khavinson, On some extremal problems of the theory of analytic functions, Moskov. Univ. Uchen. Zap. Matematika 148 (4) (1951), 133-143; English transl., Amer. Math. Soc. Transl. (2), vol. 32, Amer. Math. Soc., Providence, RI, 1963, pp. 139-154. MR0049322 (14:155f)

[N] N. K. Nikol'skiǔ, Treatise on the shift operator. Spectral function theory, Grundlehren Math. Wiss., vol. 273, Springer-Verlag, Berlin, 1986. MR0827223 (87i:47042)

[Pe1] V. Peller, Approximation by analytic operator-valued functions, Harmonic Analysis and Operator Theory (Caracas, 1994), Contemp. Math., vol. 189, Amer. Math. Soc., Providence, RI, 1995, pp. 431-448. MR1347029 (96i:47048)

[Pe2] - Hankel operators and their applications, Springer-Verlag, New York, 2003. MR1949210 (2004e:47040)

[PT1] V. V. Peller and S. R. Treil, Superoptimal singular values and indices of infinite matrix functions, Indiana Univ. Math. J. 44 (1995), 243-255. MR.1336440 (96c:47019)

[PT2] - Approximation by analytic matrix functions: The four block problem, J. Funct. Anal. 148 (1997), 191-228. MR.1461500 (98h:47021)

[PT3] — Very badly approximable matrix functions, Selecta Math. New Ser. 11 (2005), 127-154. 
[PY1] V. V. Peller and N. J. Young, Superoptimal analytic approximations of matrix functions, J. Funct. Anal. 120 (1994), 300-343. MR.1266312 (94m:47030)

[PY2] _ Superoptimal singular values and indices of matrix functions, Integral Equations Operator Theory 20 (1994), 350-363. MR.1299893 (95i:47051)

[Po] S. J. Poreda, A characterization of badly approximable functions, Trans. Amer. Math. Soc. 169 (1972), 249-256. MR0306510 (46:5636)

[T] S. R. Treil, On superoptimal approximation by analytic and meromorphic matrix-valued functions, J. Funct. Anal. 131 (1995), 386-414. MR1345037 (96g:47011)

[V] V. I. Vasyunin, Formula for multiplicity of contractions with finite defect indices, Toeplitz Operators and Spectral Function Theory, Oper. Theory Adv. Appl., vol. 42, Birkhäuser, Basel, 1989, pp. 281-304. MR.1030054 (91c:47019)

Department of Mathematics, Michigan State University, East Lansing, Michigan 48824

E-mail address: peller@math.msu.edu

Department of Mathematics, Brown University, Providence, Rhode Island 02912

E-mail address: treil@math.brown.edu

Received 30/NOV/2004

Originally published in English 